Abstracts*)

\title{
Israel Society for Auditory Research (ISAR) 2013 Annual Scientific Conference
}

\author{
Sackler School of Medicine, Tel Aviv University, Tel Aviv, Israel \\ October 8, 2013
}

\begin{abstract}
ISAR Scientific Committee
Prof. Karen Avraham (President), Prof. Joseph Attias (Treasurer), Dr. Cahtia Adelman, Dr. Karen Banai,

Dr. Yael Henkin, Prof. Liat Kishon-Rabin, Prof. Michal Luntz, Dr. Ronen Perez
\end{abstract}

\section{ISAR Conference Organizing Committee}

Dr. Karen Banai, Dr. Yael Henkin, Prof. Karen Avraham

\begin{abstract}
Special Issue on Auditory Research of the Journal of Basic and Clinical Physiology and Pharmacology

Guest Co-editors: Prof. Karen Avraham, Prof. Haim Sohmer
\end{abstract}

*These abstracts have been reproduced directly from the material supplied by the authors, without editorial alteration by the staff of this Journal. Insufficiencies of preparation, grammar, spelling, style, syntax, and usage are the authors' responsibility. 


\section{Program}

09:00-09:30 Registration and Reception

Session I: Learning and plasticity in normal and expert auditory systems Chair: Haim Sohmer

9:30 - 10:00 Plenary speaker: Andrej Kral

Cross-modal plasticity: general concept

10:00 - 10:15 N. Amir, E. Globerson, S. Levi, A. Peled, L. Kishon-Rabin

Pitch, intensity, duration and spectrum- psychoacoustic thresholds of musicians compared to non-musicians

$\begin{array}{ll}\text { 10:15-10:30 Norev and H. Pratt } & \text { Speech is special: Perceiving the very same stimuli as speech and non-speech modifies brain activity }\end{array}$

10:30 - 10:45 K. Banai and R. Yifat

Implicit online auditory learning-a domain general factor in preschool oral and written language?

11:00 - 11:15 L. Kishon-Rabin, S. Cohen, S. Ferman, D. Ari-Even Roth

11:15-11:30 D. Reznik, Y. Henkin, N. Schadel N., R. Mukamel

Lateralized enhancement of auditory cortex activity and increased sensitivity to self-generated sounds

Session II: The mechanics of hearing: ears, bones and genes

Chair: Michal Luntz

11:30-11:45 S. Chordekar, C. Adelman, H. Sohmer

11:45-12:00 $\quad$ The non-osseous (soft tissue) component of bone conduction thresholds

Histopathology of human cochlear implanted temporal bones

12:00-12:15 A. Rudnicki, S. Shivatzki, L. A. Beyer, Y. Takada, Y. Raphael and K. B. Avraham

MicroRNAs are involved in the inflammatory response in the inner ear

$12: 15-12: 30$

$12: 30-13: 00$

Business Meeting

$13: 00-14: 00$

Lunch

Posters (coffee $\&$ dessert)

Y. Bhonker, T. Elkan-Miller, F. Matsuzaki, and K. B Avraham

GPSM2/LGN and planar cell polarity in the mammalian inner ear

Y. Braver, D. Nesher, Y. Lavner and K.Banai

Practice versus exposure in the perceptual learning of time compressed speech and its adaptation

Z. Brownstein, A. Abu Rayyan, K. Ushakov, N. Idan, N. Neuberg, R. Kohen, D. Karfunkel, D. Dweik, Y. Shapira, M. Wolf, S. Shalev, B. Davidov, M. Shohat, D. Lev, S. Lieberman, E. Levy-Lahad, M. Frydman, N. Shomron, M. Kanaan and K. B. Avraham

Next-generation sequencing reveals new genes for hereditary deafness in the Middle East

H. Hassid, R. Kleinman, L. Fostick

Speech perception in different conditions

Y. Levit-Navat, R. Taitelbaum-Swead, M. Himmelfarb

Failure on first otoacoustic emissions neonatal hearing screening among twins

K. Ushakov, A. Rudnicki, O. Isakov, N. Shomron and K. B. Avraham

Regulation by novel microRNAs in the mouse inner ear

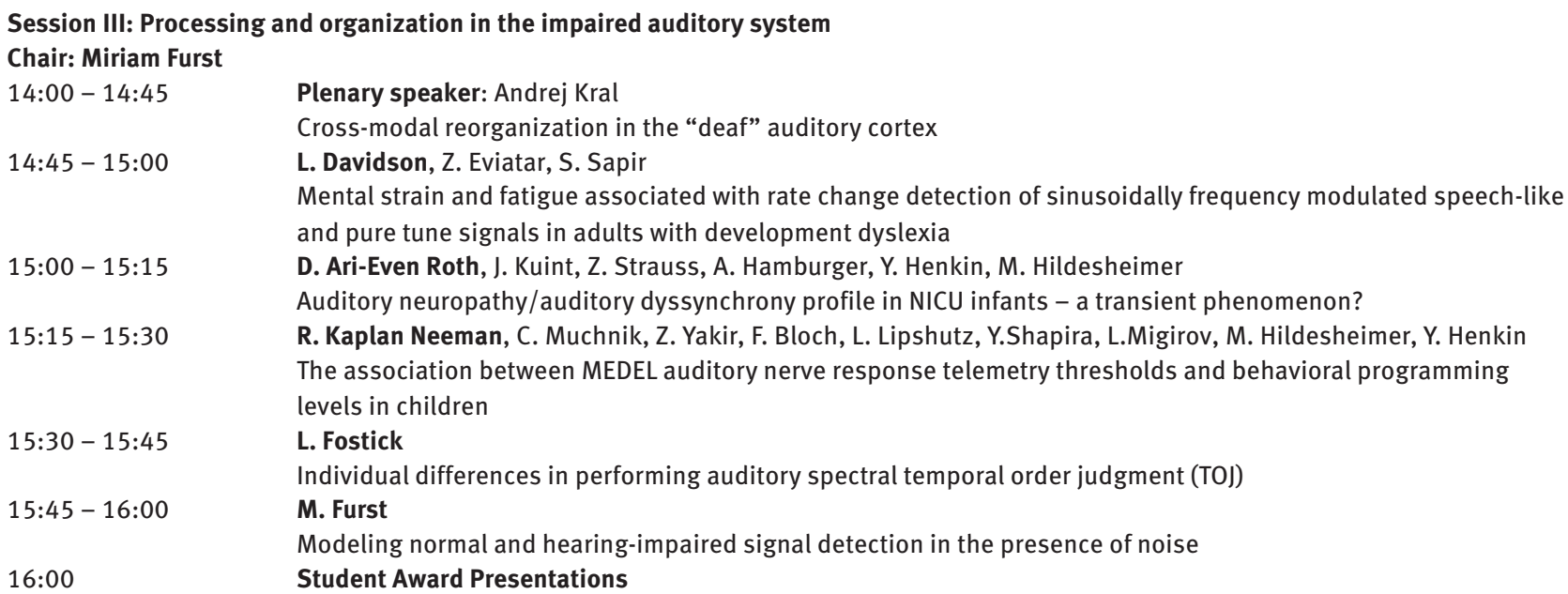




\section{PITCH, INTENSITY, DURATION AND SPECTRUM- PSYCHOACOUSTIC THRESHOLDS OF MUSICIANS COMPARED TO NON-MUSICIANS}

\author{
N. Amir ${ }^{1}$, E. Globerson ${ }^{2}$, S. Levi ${ }^{1}$, A. Peled ${ }^{1}$ and \\ L. Kishon-Rabin ${ }^{1}$ \\ ${ }^{1}$ Department of Communication Disorders, Sackler Faculty \\ of Medicine, Tel Aviv University, Tel Aviv, Israel; $2 J e r u s a l e m$ \\ Academy of Music and Dance, Jerusalem, Israel
}

Auditory performance of musicians is of interest because their exceptional listening abilities may serve as a reference for the limits of the human auditory system. Superior auditory performance of musicians has been reported primarily on tests that reflect specific facets of music, which may be the result of many years of musical training. Only a few studies, however, attempted to compare the auditory abilities of musicians and nonmusicians on basic psychoacoustic tasks. So far comparison between the two groups focused primarily on pitch discrimination abilities. The purpose of the present study was to compare the capabilities of musicians and non-musicians on different psychoacoustic abilities. Twelve musicians and 15 non-musicians, all with normal hearing, participated in this study. Each participant performed four psychoacoustic tests, obtaining thresholds for pitch, duration, intensity and spectral discrimination. For each test, threshold measure was determined as the average of 5 consecutives threshold estimates obtained using forced-choice adaptive procedures. On average musicians performed significantly better on all but the intensity task. Their continued improvement within the study suggests that the limits of the auditory system were not reached. Our findings that musicians performed better than non-musicians on tasks that they did not specifically train on may indicate the influence of top-down processing driven by music exposure and learning.

\section{AUDITORY NEUROPATHY/AUDITORY DYSSYNCHRONY PROFILE IN NICU INFANTS - A TRANSIENT PHENOMENON?}

D. Ari-Even Roth ${ }^{1,2}$, J. Kuint ${ }^{3}$, Z. Strauss ${ }^{3}$, A. Hamburger ${ }^{1,2}$, Y. Henkin ${ }^{1,2}$ and M. Hildesheimer ${ }^{1,2}$

${ }^{1}$ Hearing, Speech and Language Center, Chaim Sheba Medical Center, Tel Hashomer, Israel; ${ }^{2}$ Department of Communication Disorders, Sackler Faculty of Medicine, Tel Aviv University, Tel Aviv, Israel; 'Bepartment of Neonatology, Edmond and Lily Safra Children's Hospital, Tel Aviv, Israel;
Sackler Faculty of Medicine, Tel Aviv University, Tel Aviv, Israel; Chaim Sheba Medical Center, Tel Hashomer, Israel

The Joint Committee on Infant Hearing (2007) recommended that infants admitted to the Neonatal Intensive Care Unit (NICU) for more than five days be tested using automated auditory brainstem responses (A-ABR) as part of their screening protocol in order not to miss infants with auditory neuropathy spectrum disorder (ANSD). Berg et al. (2005) reported an incidence of $24 \%$ of NICU infants with "auditory neuropathy/auditory dyssynchrony (AN/AD) profile” i.e. passed otoacoustic emissions testing successfully and failed A-ABR on screening prior to discharge. It is not clear, however, how many of these cases represent auditory immaturity, transient neurologic abnormality or ANSD. The aims of the present study were to evaluate (1) the incidence of "AN/AD profile" prior to discharge from NICU and (2) the incidence of cases diagnosed with ANSD on followup. The screening results and audiological follow-up including diagnostic ABR of all infants born between 1.1.2010-31.7.2012 and admitted to the NICU for more than five days at the Sheba Medical Center $(\mathrm{n}=1013)$ were retrospectively examined. Results show that $20 \%$ of NICU infants $(n=207)$ demonstrated "AN/AD profile" prior to discharge. Of these, ABR remained abnormal in $6 \%$ $(n=10)$ despite normal thresholds and only one infant was diagnosed with ANSD at follow-up one month following discharge. Our data suggest that an "AN/AD profile" may be transient and emphasizes the importance of audiological follow-up in such cases.

\section{IMPLICIT ONLINE AUDITORY LEARNING-A DOMAIN GENERAL FACTOR IN PRESCHOOL ORAL AND WRITTEN LANGUAGE?}

K. Banai and R. Yifat

Department of Communication Sciences and Disorders, University of Haifa, Haifa, Israel

The role of domain general factors in the development of oral and written language in the preschool years is not well understood, but neuroconstructivist approaches to cognitive development suggest that the contribution of domain-general factors should be more salient prior to the acquisition of domain-specific knowledge in each domain. We have conducted a series of studies in preschool and kindergarten children $(n>200)$ to determine (1) if online implicit learning of stimulus regularities contributes to performance across a range or tasks - frequency 
discrimination, rapid naming, auditory memory and phonological awareness, and (2) whether such learning accounts for unique variance across a range of language and early reading tasks - phonological awareness, letter knowledge, vocabulary and verbal categorization. Online learning has been observed in all tasks tested. Furthermore, online learning accounted for up to $10 \%$ of the variance in the independent assessment of literacy related skills. We conclude that implicit online learning appears to be a domain general factor that contributes to both oral language and early reading in the preschool years. Whether this contribution diminishes once domain-specific reading-related knowledge is acquired remains to be determined in future studies.

\section{GPSM2/LGN AND PLANAR CELL POLARITY IN THE MAMMALIAN INNER EAR}

Yoni Bhonker ${ }^{1}$, Tal Elkan-Miller ${ }^{1}$, Fumio Matsuzaki ${ }^{2}$ and Karen B. Avraham ${ }^{1}$

${ }^{1}$ Department of Human Molecular Genetics and Biochemistry, Sackler Faculty of Medicine and Sagol School of Neuroscience, Tel Aviv University, Tel Aviv, Israel; ${ }^{2}$ Laboratory for Cell Asymmetry, RIKEN Center for Developmental Biology, Kobe, Japan

The planar cell polarity (PCP) pathway controls the polarization of stereocilia in the inner ear, an essential process for hearing. The kinocilium, a primary cilium in hair cells, and the basal body, a membrane-docked centriole that anchors the kinocilium, have been implicated as key players in intrinsic polarity determination in the hair cells.

A pathogenic mutation in the gene GPSM2 (also called $L G N$ ) was found in a Palestinian family with hearing loss and brain malformations (Walsh et al. AJHG 2010; Doherty et al. AJHG 2012). GPSM2 has been extensively studied in the developing brain, and is involved in mitotic spindle orientation. Through binding of NuMA and a Goi subunit, it orients the mitotic spindle and determines symmetric or asymmetric cell divisions. In GPSM2 mutants the plane of division is randomized (Konno et al. Nat Cell Biol 2008).

The role of GPSM2 in the inner ear remains unknown. We are interested in investigating its potential role in PCP and understanding the mechanism by which it affects the development of the hair bundle. Expression of this gene in the mouse cochlea was consistent with expression of PCP genes. Scanning electron microscopy of GPSM2-defective inner ears showed rotated and/or disorganized stereocilia at post-natal day 30. These results suggest that GPSM2 is involved in inner ear development.

\section{PRACTICE VERSUS EXPOSURE IN THE PERCEPTUAL LEARNING OF TIME COMPRESSED SPEECH AND ITS ADAPTATION}

\section{Y. Braver ${ }^{1}$, D. Nesher ${ }^{1}$, Y. Lavner ${ }^{2}$ and K. Banai ${ }^{1}$}

${ }^{1}$ Department of Communication Sciences and Disorders, University of Haifa, Haifa, Israel; ${ }^{2}$ Department of Computer Science, Tel-Hai College, Tel Hai, Israel

Listening to time-compressed speech, an artificial form of rapid speech, results in perceptual learning of the listened-to stimuli and in the generalization of learning to new ones. Nevertheless, whether the duration of the listening experience differentially influences learning and generalization is unknown. Here we asked whether training on 300 time-compressed sentences results in learning/ generalization in addition to what is observed following one or two sessions of brief exposure. Fifty-five listeners were divided to 3 groups: (1) naïve listeners (3 subgroups of 10 listeners), each tested on a series of 20 sentences; (2) controls $(n=15)$ that participated in a pre-test in which they transcribed a series of 20 sentences and a post-test in which they transcribed the same sentences again as well as sentences presented by a different speaker and a new set of sentences; (3) trained listeners $(n=10)$ that participated in the same pre- and post-tests as the controls as well as in a training session in which they had to verify the semantic plausibility of 300 adaptively-compressed sentences. Both learning and generalization were found to increase with increased levels of exposure. Therefore, these data suggest that training on time-compressed speech induces more learning than brief (albeit active) exposure.

\section{NEXT-GENERATION SEQUENCING REVEALS NEW GENES FOR HEREDITARY DEAFNESS IN THE MIDDLE EAST}

Z. Brownstein ${ }^{1}$, A. Abu Rayyan², K. Ushakov ${ }^{1}$, N. Idan', N. Neuberg ${ }^{1}$, R. Kohen ${ }^{1}$, D. Karfunkel ${ }^{1}$, D. Dweik ${ }^{2}$, Y.Shapira ${ }^{3}$, M. Wolf ${ }^{1,3}$, S. Shalev ${ }^{4,5}$, B. Davidov ${ }^{6}$, M. Shohat ${ }^{1,6}$, D. Lev', S. Lieberman ${ }^{8}$, E. Levy-Lahad ${ }^{8,9}$, M. Frydman ${ }^{1,10}$, N. Shomron ${ }^{11}$, M. Kanaan ${ }^{2}$ and K. B. Avraham ${ }^{1}$

${ }^{1}$ Department of Human Molecular Genetics and Biochemistry, Sackler Faculty of Medicine, Tel Aviv University, 
Tel Aviv, Israel; ${ }^{2}$ Department of Biological Sciences, Bethlehem University, Bethlehem, Palestinian Authority; ${ }^{3}$ Department of Otolaryngology, Head \& Neck Surgery, Sheba Medical Center, Tel Hashomer, Israel; ${ }^{4}$ Genetics Institute, Ha'Emek Medical Center, Afula, Israel; ${ }^{5}$ Rappaport Faculty of Medicine, Technion-Israel Institute of Technology, Haifa, Israel; 'Department of Medical Genetics, Rabin Medical Center, Petah Tikva, Israel; 'Institute of Medical Genetics, Wolfson Medical Center, Holon, Israel; ${ }^{8}$ Medical Genetics Institute, Shaare Zedek Medical Center, ${ }^{9}$ Hebrew University Medical School, Jerusalem, Israel; ${ }^{10}$ Danek Gartner Institute of Human Genetics, Sheba Medical Center, Tel Hashomer, Israel; ${ }^{11}$ Department of Cell and Developmental Biology, Sackler Faculty of Medicine, Tel Aviv University, Tel Aviv, Israel

Although over 100 genes are known to be involved in hereditary hearing loss, a significant portion of this sensory defect remains unsolved. High rates of consanguinity in the Middle Eastern population and many different ethnic groups contribute to these unsolved cases. To address this challenge, we combined targeted gene capture and next-generation sequencing (also referred to as massively parallel sequencing). A pool of 284 genes was employed, including 118 human genes, three human microRNAs, and 163 human orthologues of mouse deafness genes. DNA derived from ninety-six Israeli Jewish and Palestinian Arab patients were analyzed using the Illumina HiSeq 2000. Coordination with homozygosity mapping in consanguineous families optimized bioinformatics analysis. This method led to a doubling of the number of genes associated with hearing loss in the Middle East population, including novel mutations in known human deafness genes and most compelling, mutations in genes previously associated only with mouse deafness. Protein structure predictions were made to provide insight into the mechanisms leading to deafness. The discovery of novel genes and mutations has implications worldwide, as many mutations first found in the Middle East have turned out to be present in other populations. This strategy allows for improved diagnostics in an economically and temporally feasible manner, and establishing etiologically-based genetic counseling and hearing loss management.

\section{THE NON-OSSEOUS (SOFT TISSUE) COMPONENT OF BONE CONDUCTION THRESHOLDS}

\author{
S. Chordekar' ${ }^{1}$ C. Adelman ${ }^{2,3}$ and H. Sohmer ${ }^{4}$ \\ ${ }^{1}$ Department of Communication Disorders, Sackler Faculty \\ of Medicine, Tel Aviv University, Israel; ${ }^{2}$ Speech and
}

Hearing Center, Hebrew University School of MedicineHadassah Medical Center, Jerusalem, Israel; ${ }^{3}$ Department of Communication Disorders, Hadassah Academic College, Jerusalem, Israel; "Department of Medical Neurobiology (Physiology), Institute for Medical Research-Israel-Canada, Hebrew University-Hadassah Medical School, Jerusalem, Israel

In order to differentiate between the osseous component and the non-osseous (soft tissue conduction-STC) component of bone conduction (BC), the clinical bone vibrator (BV) was applied to the forehead of normal subjects, equipped with ear plugs, in two different situations: 1.500 gram application force (via headband); 2. BV applied only to a layer of ultrasound gel on the skin, without direct contact or application force to the skin. In this latter situation, it is likely that there is no osseous BC. Thresholds to pure tones at 1.0, 2.0 and $4.0 \mathrm{kHz}$ were assessed. There was no difference in threshold between these two situations. This provides additional evidence that $\mathrm{BC}$ thresholds in the clinic are actually the non-osseous STC component of BC. This conclusion also explains the clinical success of using $\mathrm{AC}$ and $\mathrm{BC}$ threshold determinations for the differential diagnosis between conductive and sensori-neural hearing losses, even though classically, it is thought that osseous BC mechanisms involve parts of the middle ear and the two windows; the non-osseous-STC component of $\mathrm{BC}$ represents pure cochlear function, without involvement of the middle ear and the two windows.

\section{MENTAL STRAIN AND FATIGUE ASSOCIATED WITH RATE CHANGE DETECTION OF SINUSOIDALLY FREQUENCY MODULATED SPEECH-LIKE AND PURE TUNE SIGNALS IN ADULTS WITH DEVELOPMENT DYSLEXIA}

\author{
L. Davidson ${ }^{1}$, Z. Eviatar ${ }^{1}$ and S. Sapir ${ }^{2}$ \\ Departments of ${ }^{1}$ Psychology and ${ }^{2}$ Communication Sciences \\ and Disorders, Haifa University, Haifa, Israel
}

Purpose. To examine learning patterns and mental strain and fatigue effects associated with rate change detection (RCD) of sinusoidally frequency modulated speech-like (FMSL) and pure tone (FMPT) signals in university students with developmental dyslexia (DD, $n=30$ ) and typical readers (TR, $\mathrm{n}=30$ ).

Methods. There were three types of stimuli, one in which the rate of signal modulation was constant, one in which the rate of modulation increased by $1 \mathrm{~Hz}$, and one in which the rate of modulation decreased by $1 \mathrm{~Hz}$ in the middle of the stimulus. The initial rate of modulation 
was manipulated to vary RCD difficulty. Each subject performed 6 identical runs, and in each run, the highest level of RCD was determined. Hart rate variability (HRV) was used to measure mental effort, and a visual analog scale (VAS) to measure self-rated fatigue.

Main Findings. The DD group showed significant decrement in performance, and increased mental strain and fatigue. The TR group showed significant improvement in performance, a decrease in mental effort, and an increase in fatigue. There was a significant correlation between the RCD performance and fatigue. These between group differences were observed mainly with the FMSL signals.

Conclusion. Students with DD may have difficulties processing speech-like signals, thus prone to mental fatigue.

\section{INDIVIDUAL DIFFERENCES IN PERFORMING AUDITORY SPECTRAL TEMPORAL ORDER JUDGMENT (TOJ)}

\section{Fostick}

Department of Communication Disorders, Ariel University, Ariel, Israel

Background. Auditory spectral TOJ measures the ability to perceive the order of two sounds, differing in frequency. Hirsh (1959) and Hirsh and Serrick (1961) showed that the SOA threshold needed for this task was $17 \mathrm{msec}$ regardless of the stimuli characteristics. Using $1 \mathrm{kHz}$ and $1.8 \mathrm{kHz}$ tones, we found that $50 \%$ percent of participants had very low thresholds ( $<5 \mathrm{msec}) ; 23 \%$ had thresholds ranging from $5-120 \mathrm{msec}$ and $27 \%$ had very high thresholds $(>120 \mathrm{msec})$. The current study focuses on characterizing this pattern of response.

Methods. In a series of studies conducted on 461 participants, we measured the effect of psychoacoustic methods, tone frequency, participant characteristics, and linguistic and cognitive abilities.

Results. Methodology, hearing level, temporal resolution thresholds, speech perception, auditory and spatial memory, attention, and bilingualism had no effect on the pattern of threshold distributions. Low thresholds $(<5 \mathrm{msec})$ were more prevalent when the stimuli were low frequency tones $(300$ and $600 \mathrm{~Hz}$ ) and less prevalent when the tones were $1 \mathrm{kHz}$ and $3.5 \mathrm{kHz}$. High spectral TOJ thresholds $(<120 \mathrm{msec})$ were associated with poorer scores on the matrices and phonological awareness task.

Conclusions. Auditory spectral TOJ might not measure temporal resolution, but rather an ability that is more related to sensitivity to speech sounds.

\section{MODELING NORMAL AND HEARING-IMPAIRED SIGNAL DETECTION IN THE PRESENCE OF NOISE}

\section{Furst}

Department of Engineering, Tel Aviv University, Tel Aviv, Israel

Psychoacoustical investigations have demonstrated that signal detection is affected by existence of distracting noise. The minimal perceived signal-to-noise ratio (SNR) is much larger in the hearing impaired relative to normal hearing people. Another psychoacoustical phenomenon that correlates to perception in noise is the co-modulation masking release (CMR). It describes the reduced masking of a pure tone when the masking is a noise that is coherently amplitude modulated (comodulated) over the total range of the spectrum compared to masking by an unmodulated noise of the same bandwidth and overall energy. In general CMRs in the monaural condition were smaller for hearing-impaired than for normal ears. A computational model was developed in order to demonstrate the difference between normal and hearing impaired listeners. The model includes a complete cochlear model with integrated outer hair cells and tectorial membrane; an inner hair cell-synapse model that transduce the cilia motion to auditory nerve (AN) instantaneous rate. The AN activity is considered as a random point process, and particularly as a non-homogeneous Poisson process (NHHP). On basis of this model we have derived human's performance in just-noticeable (JND) experiments in which amplitude discrimination in presence of noise was involved. We have successfully predicted major CMR properties as a function of frequency and noise properties for normal and impaired auditory system.

\section{HISTOPATHOLOGY OF HUMAN COCHLEAR IMPLANTED TEMPORAL BONES}

O. Handzel

Cochlear Implant Program, Department of Otolaryngology/ Head and Neck Surgery, Tel Aviv Sourasky Medical Center (Ichilov), Tel Aviv, Israel; Sackler Faculty of Medicine, Tel Aviv University, Tel Aviv, Israel

Background: Cochlear implants (CI) can enable patients to regain the use of lost sense of audition. The relationship between the implant, inner ear, central processes, and their implications on post implantation success has not been completely elucidated. 
Goal: Describe the role of histopathology of human cochlear implanted temporal bones as a tool to answer some of the above detailed questions.

Methods: Human temporal bones from donors implanted during life and processed post mortem were analyzed using various light microscopy stains. Three-dimensional reconstruction with propriety software can be helpful in some cases.

Results: The relationship between the status of the peripheral auditory system and CIs will be discussed. The effect of residual neuronal structures on the outcome of implantation and vice versa-the effect of implantation on the organ of Corti, lateral cochlear wall and spiral ganglion cells will be described. In addition, the effect of implantation on the vestibular system will be elucidated as well.

Conclusion: Histopathology of cochlear-implanted temporal bones can be a powerful tool to study the relationship between the implant the peripheral auditory and vestibular system. This mode of research can provide data not obtainable by any other research techniques.

\section{SPEECH PERCEPTION IN DIFFERENT CONDITIONS}

H. Hassid ${ }^{\star}$, R. Kleinman ${ }^{\star}$ and L. Fostick ${ }^{\star}$ Equal contribution Department of Communication Disorders, Ariel University, Ariel

Background. Speech perception is a measure of hearing sensitivity, temporal perception, and cognitive ability. The literature is heterogeneous regarding speech perception tasks. In the present study we compared performance on speech perception in white noise (WN) in three conditions: continuous noise (CN), noise per word $(\mathrm{NpW})$, and noise per words with preceding sentence (NpWwS).

Methods. Twenty-four students performed the Hebrew version of the AB test (Boothroyd, 1984), accompanied with $\mathrm{WN}, \mathrm{SNR}=0$. In the $\mathrm{CN}$ condition, the noise started three seconds before the first word and continued nonstop until the end of the list. In the NpW, the noise started at the beginning of each word and stopped when the word ended, leaving periods of silence between the words. The NpWwS condition was similar to the NpW condition, but each word was preceded with the sentence "please say..." which was presented in quiet.

Results. Significant condition effect was found $\left(\mathrm{F}_{(2,46)}=3.07\right.$, $\mathrm{p}=.05)$ with $\mathrm{CN}$ condition revealing a higher accuracy rate (62.5\%) than NpW (57.9\%) and NpWwS (58.3\%), which did not differ.
Discussion. CN was the easiest condition, probably due to adaptation. Nevertheless, this condition might simulate better natural listening conditions that are usually done with continuous noises. In this regard, this study should be repeated with babble or cafeteria noises.

\section{SPEECH IS SPECIAL: PERCEIVING THE VERY SAME STIMULI AS SPEECH AND NON-SPEECH MODIFIES BRAIN ACTIVITY}

N. Horev and H. Pratt

Evoked Potentials Laboratory, Technion-Israel Institute of Technology, Haifa, Israel

Sine-wave speech (SWS), in which speech formants are replaced by 3 tones, can be perceived as either speech or non-speech, depending on listeners' expectations about the nature of the stimuli. SWS thus allows comparing brain activity to speech and non-speech sounds using the same acoustic stimuli. Auditory event-related potentials (ERPs) were recorded from $61 \mathrm{scalp}$ electrodes during active discrimination of SWS stimulus pairs using spectral ( $\mathrm{ubu} / \mathrm{udu}$ ) or temporal (ubu/upu) cues. In a first session, subjects discriminated between SWS stimuli, unaware of their speechlike nature considering them non-speech. In the second session, the same subjects performed the same discrimination task after learning to perceive the SWS as speech. ERPs waveforms to the first stimulus in the pairs were analyzed and source current density estimation was conducted. Perceiving the very same stimuli as speech or non-speech was associated with significant voltage differences around the main scalp recorded ERPs peaks. Source estimation revealed higher current density in auditory cortices to "speech" stimuli during early (around $230 \mathrm{~ms}$ ) processing, and higher current density to "non-speech" stimuli later (340-500 ms) times. Differences were significant mainly within the right superior temporal cortex and the right parieto-temporal junction. These results support distinct "speech" and "nonspeech" modes of auditory processing.

\section{THE ASSOCIATION BETWEEN MED-EL AUDITORY NERVE RESPONSE TELEMETRY THRESHOLDS AND BEHAVIORAL PROGRAMMING LEVELS IN CHILDREN}

R. Kaplan Neeman ${ }^{1,3}$, C. Muchnik ${ }^{1,3}$, Z. Yakir ${ }^{1}$, F. Bloch ${ }^{1}$, L. Lipshutz ${ }^{1,}$ Y.Shapira ${ }^{2}$, L.Migirov ${ }^{2}$, M. Hildesheimer ${ }^{1,3}$ and Y.Henkin ${ }^{1,3}$

${ }^{1}$ Hearing, Speech and Language Center and ${ }^{2}$ Department of Otolaryngology, Head and Neck Surgery, Sheba Medical 
Center, Tel Hashomer, Israel; ${ }^{3}$ Department of Communication Disorders, Sackler Faculty of Medicine, Tel Aviv University, Tel Aviv, Israel

The difficulty in obtaining thresholds and comfortable levels in young children with cochlear implants has led to the development of electrophysiological measures for predicting these parameters. The most widely used electrophysiological measure used for this purpose is the electrically evoked compound action potential (ECAP). Numerous studies have determined the associations between ECAPs and thresholds (Tlevels) and comfortable levels (C/Mlevels) obtained behaviorally from Cochlear and Advanced Bionics recipients. Data concerning ECAP measurements and their relationship with programming levels in children using the MED-EL device is rare. The objective of the present study was, therefore, to explore the association between Auditory Nerve Response Telemetry (ART) measurements and programming levels in children using the MED-EL device. For this purpose we studied ART thresholds and behaviorally obtained most comfortable levels (M) in a group of children ranging from 18 months to 12 years, with age at implantation of 10-48 months that were using their implants for at least six months. Post-operative ART thresholds and behavioral $\mathrm{M}$ levels were compared at basal, medial and apical electrodes. Initial results showed that ART responses were elicited in $97 \%$ of tested electrodes. Furthermore, t-ART fell at $70-75 \%$ of the dynamic range. These results coincide with previous findings obtained from children using Cochlear and Advanced Bionics devices, and indicate that ART measurements can assist in the programming of young children using the MED-EL device.

\section{LEARNING AN INVENTED AUDITORY NON-LINGUISTIC RULE: DO CHILDREN AND ADULTS USE SIMILAR MEMORY- BASED MECHANISMS?}

L. Kishon-Rabin, S. Cohen, S. Ferman, D. Ari-Even Roth Department of Communication Disorders, Sackler Faculty of Medicine, Tel Aviv University, Tel Aviv, Israel

Learning linguistic rules (e.g., grammar) by children is assumed to involve implicit memory-based learning mechanisms. This learning continues through school years and adulthood and is then supported by explicit learning. The present study was conducted to answer the following questions: (1) Are learning processes specific to language learning or are they part of common mechanisms that underlie learning of rules with nonlinguistic stimuli? (2) Do children differ from adults in their learning of rules when using nonlinguistic stimuli? Ten young adults and 10 children participated in 5 learning sessions. In each session, participants listened twice to a modeling list of 16 sequences that each consisted of the same four different non-speech sounds but in a different order according to an invented rule. Listeners were asked to judge sequences (correct or incorrect) and were required to complete the final sound of a sequence. The mean group data show significant learning of the invented rule in both tasks for the adults but only in the completion task for the children, although large inter-subject variability was observed. Our data suggest the following: (1) the completion task represents a more automatic type of learning compared to the judgment task, (2) children rely more on implicit than on explicit learning processes whereas adults use both and (3) a common mechanism may underlie learning of linguistic and non-linguistic rules.

\section{CROSS-MODAL PLASTICITY: GENERAL CONCEPT}

\section{A. Kral}

Institute of Audioneurotechnology \& Dept. of Experimental Otology, ENT Clinics,Hannover School of Medicine, Hannover, Germany

Deprivation in a sensory modality, particularly if the onset is early in development, leads to compensational changes in the other sensory system. Both visual and auditory deprivations are known to reorganize spared sensory system at both the neuronal and behavioral level. The consequences are supranormal and subnormal performance in the spared modalities. According to deficiency theories, deprivation negatively affects spared modalities, as each existing modality provides a particular informational structure that is used and referenced to by other sensory modalities. According to compensation theories, deprivation leads to supranormal performance in spared modalities, as these can make use of the neuronal resources that would be otherwise used by the deprived modality. The auditory system may have a particular role in temporal order and sequencing, the visual system in spatial relations and localization.

The neuronal mechanisms of supranormal performance include bottom-up, lateral and top-down mechanisms. They include growth of new connections, strengthening of existing interneuronal connections and also reorganization in the use of brain regions and their recruitment in attentional processes. The talk will review existing evidence cross-modal reorganization in the brain with a particular emphasis on auditory deprivation in 
development and adulthood. It will discuss methodological limitations in both animal and human studies of the topic.

Supported by Deutsche Forschungsgemeinschaft (DFG Kr 3370/1-3 and Cluster of Excellence Hearing4All).

\section{CROSS-MODAL PLASTICITY IN THE “DEAF” AUDITORY CORTEX}

A. Kral

Institute of Audioneurotechnology \& Dept. of Experimental Otology, ENT Clinics, Hannover School of Medicine, Hannover, Germany

Congenital and early deafness reorganizes the auditory system and leads to supranormal abilities in other sensory systems. Congenitally deaf cats (CDCs) demonstrate supranormal performance in the visual domain (detection and localization in the periphery and movement detection. Furthermore, cooling deactivation localized the site of reorganization into secondary fields dorsal zone (DZ) and posterior auditory field. The field A1 (primary auditory field) and AAF were not involved in such reorganization. Application of retrograde tracer into DZ and A1 demonstrated a differential recruitment of these fields in cross-modal reorganization; whereas A1 did not show significant cortical cross-modal reorganization in CDCs, field DZ showed visual and somatosensory projections not observed in hearing controls.

In follow-up experiments, recordings were taken from field DZ and an adjoining visual fields (anterior and posterior MLS) under cochlear implant (auditory) and visual stimulation in precisely-controlled isoflurane/ $\mathrm{N}_{2} \mathrm{O}$ anesthesia in 8 cats ( 4 hearing, 4 deaf). Altogether, 1424 unit responses were evaluated, $\sim 700$ in each group. Spontaneous activity was significantly higher in MLS than in DZ in both groups of animals. Unit responses showing statistically significant correlation with current level were considered responsive to auditory stimulation. There were significant auditory responses in both groups of animals in similar proportion of units in DZ. The present results suggest a modest visual crossmodal reorganization of field DZ in congenital deafness. However, the auditory responsiveness was preserved in this field as well. The extent of the effect suggests that also other (possibly attentional) processes are involved in behavioral supranormal visual performance in motion detection.

Supported by Deutsche Forschungsgemeinschaft (DFG Kr 3370/1-3 and Cluster of Excellence Hearing4All).

\section{FAILURE ON FIRST OTO ACOUSTIC EMISSIONS NEONATAL HEARING SCREENING AMONG TWINS}

Y. Levit-Navat ${ }^{1,2}, \quad$ R. Taitelbaum-Swead ${ }^{2}$ and M. Himmelfarb ${ }^{1,2}$

${ }^{1}$ Speech and Hearing Unit, Souraky Medical Center, Tel Aviv, Israel; ${ }^{2}$ Ariel University, Ariel, Israel

Failure on first neonatal otoacoustic emission (OAE) hearing screening was reported to occur in $5-20 \%$ of the infants and was attributed to vernix caseosa and debris in the external ear canal or middle ear fluid. The purpose of the present study was to evaluate the possible interference of twin deliveries with fail rate of the first transient evoked OAE (TEOAE) hearing-screening test. A retrospective analysis of the newborn hearing-screening database of all the neonates born during 2012 was conducted. The number of repeated TEOAE tests was compared between singletons and twins. Of 11,188 live newborns, 519 (4.6\%) were twins. Repeated TEOAE tests were performed on 815 (7.64\%) of singletons, and on 58 (11.1\%) of the twins. A higher rate of repeated screening tests was found among second-born twins (13\%) than in first-born twins (9.2\%). A higher fail rate on the first TEOAE screening hearing test was found among twins as compared to singletons and was even higher among second-born twins. The secondborn twin is more likely to be intubated, have respiratory distress syndrome, need resuscitation and have lower Apgar scores. It is possible that these babies may suffer from delayed middle ear fluid resorption impeding recording of OAE responses.

\section{LATERALIZED ENHANCEMENT OF AUDITORY CORTEX ACTIVITY AND INCREASED SENSITIVITY TO SELF-GENER- ATED SOUNDS}

D. Reznik ${ }^{1,2}$, Y. Henkin ${ }^{3,4}$, N. Schadel ${ }^{1,2}$ and R. Mukamel ${ }^{1,2^{\star}}$ ${ }^{1}$ School of Psychological Sciences, Tel Aviv University, Tel Aviv, Israel; ${ }^{2}$ Sagol School of Neuroscience, Tel Aviv University, Tel Aviv, Israel; ' 3 Department of Communication Disorders, Sackler Faculty of Medicine, Tel Aviv University, Tel Aviv, Israel; ${ }^{4} H e a r i n g, ~ S p e e c h$, and Language Center, Sheba Medical Center, Tel Hashomer, Israel

Performing an action with auditory consequences (like pressing a button to ring the door-bell) modulates the response in the auditory cortex to an otherwise identical stimulus when passively heard. Such modulation has been suggested to occur through a corollary discharge sent from motor cortex during voluntary actions. 
However, the relationship between the effector used to generate the sound, type of modulation, and changes in perceptual sensitivity are unclear. Using fMRI, we demonstrate bilateral enhancement in the auditory cortex to selfgenerated vs. externally-generated sounds. Furthermore, this enhancement was stronger when the sound-producing hand was contralateral to the auditory cortex. At the behavioral level, binaural hearing thresholds were lower for self-generated sounds and monaural thresholds were lower when sounds were triggered by the hand ipsilateral to the stimulated ear. Together with functional connectivity analysis, our results suggest that a corollary discharge sent from active motor cortex enhances activity in auditory cortex and increases perceptual sensitivity in a lateralized fashion.

\section{MICRORNAS ARE INVOLVED IN THE INFLAMMATORY RESPONSE IN THE INNER EAR}

A. Rudnicki ${ }^{1}$, S. Shivatzki ${ }^{1}$, L. A. Beyer ${ }^{2}$, Y. Takada ${ }^{2}$, Y. Raphael $^{2}$ and K. B. Avraham ${ }^{1}$

${ }^{1}$ Department of Human Molecular Genetics and Biochemistry, Sackler Faculty of Medicine, Tel Aviv University, Tel Aviv, Israel; ${ }^{2}$ Kresge Hearing Research Institute, Department of Otolaryngology - Head and Neck Surgery, University of Michigan, Ann Arbor, MI, USA

MicroRNAs (miRNA) are small non-coding RNAs that regulate gene expression through the RNA interference (RNAi) pathway. By binding to sequences in the $3^{\prime}$ untranslated region (UTR) of genes, a miRNA can inhibit target mRNAs by translational suppression and mRNA destabilization. miRNAs play an important role in the development and regulation in the inner ear, and mutations in miRNAs lead to deafness in humans and mice. In this study, we propose that miR-224 and its target Ptx3 play an essential role in the innate inflammatory response in the inner ear. We show that miR-224 is expressed in mouse inner ear sensory epithelia. Luciferase and over-expression assays demonstrate that miR-224 targets Ptx3. In an inner ear inflammation model, LPS (lipopolysaccharide) was injected into mouse inner ears, leading to an immune response. miR-224 and
Ptx3 expression was increased in LSP-induced inflammation. We hypothesize that Ptx3 in a powerful protein in the immune cascade and needs to be subjected to tight regulation. In conclusion, we found that miR-224 directly targets Ptx3 in the NF- $\kappa$ B inflammatory pathway in inner ear LSPinduced inflammation. This finding sheds light on the role of miRNAs in the complex regulation of the inflammatory pathway in the inner ear.

\section{REGULATION BY NOVEL MICRORNAS IN THE MOUSE INNER EAR}

K. Ushakov ${ }^{1}$, A. Rudnicki ${ }^{1}$, O. Isakov ${ }^{2}$, N. Shomron ${ }^{2}$ and K.B. Avraham ${ }^{1}$

${ }^{1}$ Department of Human Molecular Genetics and Biochemistry, ${ }^{2}$ Department of Cell and Developmental Biology, Sackler Faculty of Medicine, Tel Aviv University, Tel Aviv, Israel

microRNAs (miRNAs) regulate gene expression through the RNA interference pathway. They bind to sequences at the $3^{\prime}$ untranslated region (UTR) of genes and therefore can inhibit target mRNAs by translational repression and mRNA destabilization. miRNAs play a critical role in the development and regulation of sensory systems, including the auditory system, and are known to be involved in deafness in both humans and mice. To dissect the function of miRNAs in the inner ear, we performed RNA-Seq on small RNAs from mouse inner ear sensory epithelia. Reads were aligned to the mature mouse miRNA database and miRDeep2 was used for novel miRNA prediction. From the predicted pre-miRNAs that were expressed in both cochlear and vestibular SE, we selected two miRNAs for further study, which are found in introns of the genes Tectb and Gap43. qRT-PCR and in situ hybridization confirmed the presence and spatial expression of these miRNAs in the mouse inner ear. We used bioinformatics to predict the targets of these miRNAs and verified them by in vitro overexpression and luciferase assays. Dissecting the pathways of miRNA-target interactions may shed light on the role miRNAs play in the regulation of gene expression in the auditory system and the mechanisms leading towards deafness. 